Z. klin. Chem. u. klin. Biochem.

8. Jg., S. $572-576$, November 1970

\title{
Intestinale Lactosespaltung subtotal pankreatektomierter Ratten ${ }^{1}$ )
}

\author{
Von R. Böhmer, R. Goberna und K. Rommel \\ Aus der Sektion für Kliniscle Cbemie (Doz. Dr. K. Rommel) des Zentrums für Innere Medizin und Kinderbeilkunde der \\ Universität Ulm
}

(Eingegangen am 27. Juni 1970)

Bei subtotal pankreatektomierten Ratten wurde die Lactosedigestion mit der Perfusionstechnik untersucht. Opetierte Tiere spalteten signifikant weniger Lactose als gesunde Kontrolltiere.

Pankreozymin i. v. blieb bei pankreatektomierten Tieren ohne Effekt auf die Lactosedigestion, während es bei Kontrolltieren eine signifikante Hemmung der Lactosespaltung bewirkte.

Die Ergebnisse wurden auf der Grundlage neuer Hypothesen über die hormonale Beeinflußbarkeit der Lactosedigestion und -absorption diskutiert.

\section{The intestinal bydrolysis of lactose in incompletely pancreatectomised rats}

The digestion of lactose was studied in incompletely pancreatectomised rats with the perfusion technique. Operated rats hydrolysed significantly less lactose than healthy control animals.

Pancreozymin i. v. had no effect on lactose digestion in pancreatectomised rats, while it caused a significant inhibition of lactose cleavage in the control animals. The results are discussed on the basis of new hypotheses for the influence of hormones on the digestion and absorption of lactose.

Bei früheren Untersuchungen (1) stellten wir fest, daß die Lactosedigestion im Dünndarm der Ratte durch intravenöse Gabe von Pankreozymin gehemmt wird. Es stellt sich die Frage, ob diese Hemmung ein direkter Pankreozymin-Effekt an der Mukosa des Jejunums ist oder ob es sich um einen indirekten PankreozyminEffekt infolge Stimulation des endokrinen Pankreas mit Insulinfreisetzung handelt. Zur Klärung dieser Frage wurde die Lactosedigestion an subtotal pankreatektomierten Tieren mit und ohne Pankreozymingabe untersucht.

\section{Methodik}

Männliche Wistar-Ratten wurden bei einem Körpergewicht von 80-100 g nach der von Shapiro und Pincus (2), Ingle und ThorN (3) und Fogria (4) angegebenen Methode subtotal (95\%) pankreatektomiert.

5-8 Wochen nach der Operation untersuchten wir die Lactosedigestion des Jejunums mit Hilfe der Perfusionstechnik. Nach 12 stdg. Nahrungskarenz wurden die Tiere mit $0,3 \mathrm{ml} 5$ proz. Pentobarbital (Nembutal) intraperitoneal narkotisiert. Wir perfundierten ein $10 \mathrm{~cm}$ langes Darmstück, beginnend $30 \mathrm{~cm}$ distal vom Pylorus, mit konstanter Geschwindigkeit von 0,2 $\mathrm{m} / \mathrm{Min}$.

Als Perfusionslösung diente eine isotone Natrium-Sulfat-Lösung, welche $100 \mathrm{mg} / 100 \mathrm{ml}$ Lactose und $100 \mathrm{mg} / 100 \mathrm{ml}$ Kollidon (Polyvinylpyrrolidon) als nicht absorbierbare Substanz enthielt $(\mathrm{pH}=5,1)$. Die Temperatur der Perfusionslösung wurde der Körpertemperatur des Tieres angeglichen, indem der zuführende Schlauch durch ein warmes Wasserbad geleitet wurde. Durch Zurückverlagern des Darmes in die Bauchhöhle und Bedecken mit feuchten Tüchern ( 0,9 proz. NaCl-Lösung) wurde ein Austrocknen des Darmes während des Versuches verhindert.

Das Perfusat wurde in 6 Fraktionen über jeweils 10 Min. getrennt gesammelt. Die Bestimmung der freien Galaktose und der Lactose im Perfusat erfolgte enzymatisch mit Galaktosedehydrogenase

1) Mit Unterstützung der Deutschen Forschungsgemeinschaft, Bad Godesberg.
(Testpack Boehringer, Mannheim) und $\beta$-Galaktosidase. Zunächst wurde die freie Galaktose bestimmt. Nach Stillstand der Reaktion wurden $0,02 \mathrm{ml} \beta$-Galaktosidase (Fa. Boehringer, Mannheim) hinzugefügt. Nach gutem Durchmischen wurde ebenfalls der Stillstand der Reaktion bei $366 \mathrm{~nm}$ abgewartet und die Extinktionsdifferenz $\triangle \mathrm{E}$ gemessen. Die Lactosekonzentration $(\mathrm{mg} / 100 \mathrm{~m} l)$ ergibt sich nach der Formel $\Delta \mathrm{E} \cdot 173,1=\mathrm{mg} / 100 \mathrm{~m} l$ Lactose.

Der Kollidonnachweis effolgte in Anlehnung an die von ScHUBert und Werner (5) angegebene Methode in der Modifizierung von BÖHMER Und ROMMEL (1).

Die Lactose-Digestion (\%) wurde nach der Formel

$$
\mathrm{D}(\%)=100 \cdot\left(1-\frac{\mathrm{L}_{\mathrm{P}} \cdot \mathrm{K}_{\mathrm{S}}}{\mathrm{K}_{\mathrm{P}} \cdot \mathrm{L}_{\mathrm{s}}}\right)
$$

berechnet.

$\mathrm{L}_{\mathrm{s}}=$ Lactosegehalt der Stammlösung $(\mathrm{mg} / 100 \mathrm{ml})$

$\mathrm{K}_{\mathrm{s}}=$ Kollidongehalt der Stammlösung $(\mathrm{mg} / 100 \mathrm{ml})$

$\mathrm{L}_{\mathrm{P}}=$ Lactosegehalt des Perfusates $(\mathrm{mg} / 100 \mathrm{ml}$ )

$K_{\mathbf{P}}=$ Kollidongehalt des Perfusates $(\mathrm{mg} / 100 \mathrm{~m} l$ ).

Die Untersuchungen wurden an einem Kollektiv subtotal pankreatektomierter Tiere ohne Pankreozymingabe und an einem gleichen Kollektiv nach Gabe von $6 \mathrm{E} / \mathrm{kg}$ Pankreozymin (Karolinska Institutet, Stockholm, Schweden) i. v. durchgeführt. Zum Vergleich dienten zwei Kollektive nicht operierter Tiere, deren Jejunum ebenfalls ohne und mit vorheriger Gabe von Pankreozymin perfundiert wurde.

\section{Ergebnisse}

Tábelle 1 zeigt das Verhalten der Lactosedigestion bei den vier untersuchten Kollektiven. Die erste Fraktion 0-10 Min..nach Perfusionsbeginn wurde verworfen, da aufgrund der angewendeten Perfușionstechnik dieser Wert falsch niedrig ausfällt.

Die Unterschiede der Lactose-Digestion zwischen den vier verschiedenen Kollektiven wurden mit Hilfe des STUDENT-t-Testes auf ihre statistische Signifikanz untersucht (Tab. 2). Aus Tabelle 2 ist zu ersehen, daß die Ver- 


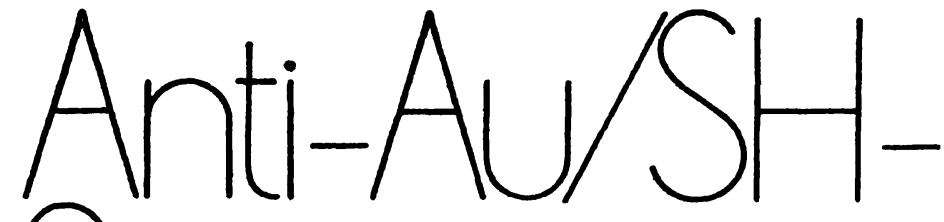

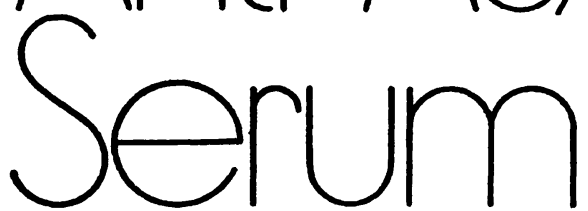

der Behringwerke

zum Nachweis des Au/SH (Australla/Serumhepatitis)-Antigens mit Milrro-Ouchterlony-Test oder Überwanderumgselektrophorese. 


\section{Hohe Genauigkeiten erfordern größte Aufmerksamkeit. Besser überläßt man das geeigneten Meß- und Dosiergeräten.*}

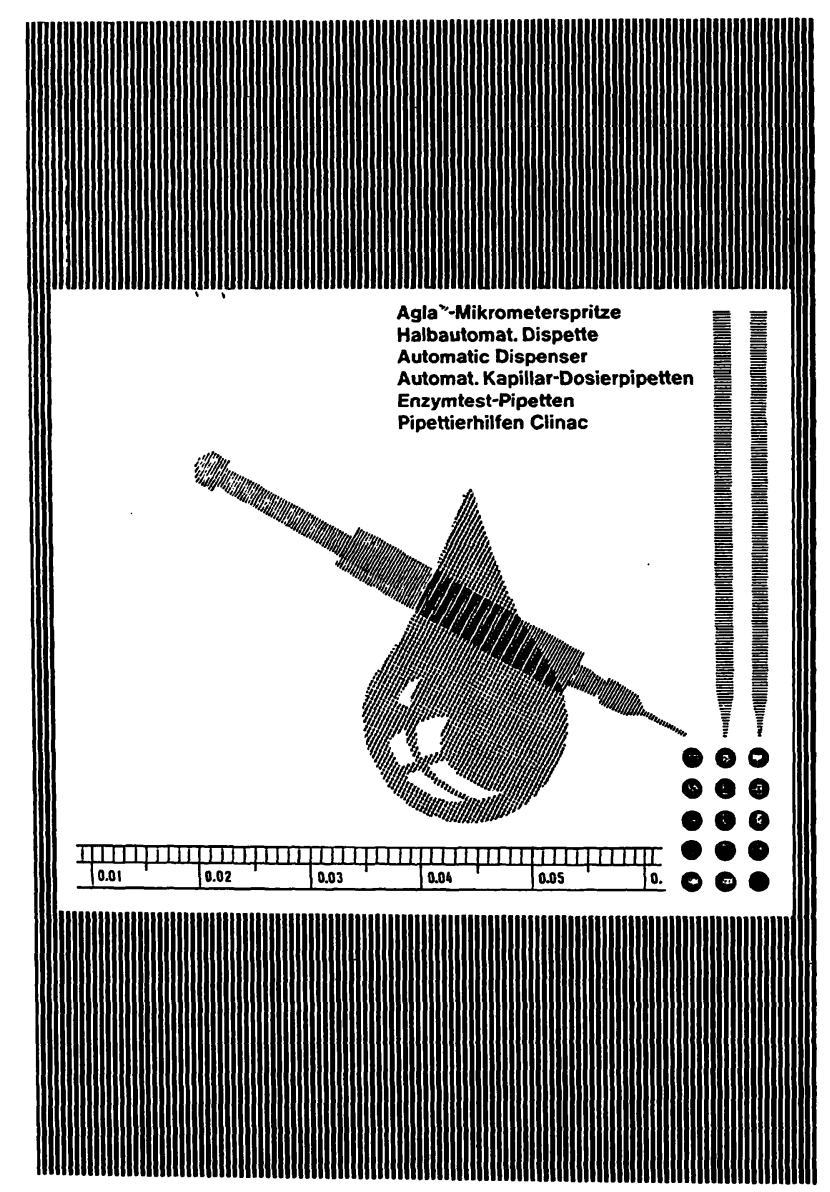

* schon aus Gründen des Zeitgewinns

\section{Unsere interessante Druckschrift MD-708 10 informiert Sie ausführlich.} 6900 Heidelberg 1, Postf. 750, Tel. 06221/20045

\section{DESAGA Katalog 70}

Diese Neuerscheinung informiert Sie über das wohl breiteste Apparateprogramm auf dem Gebiet der Trenntechnik; kurz, prägnant und in übersichtlicher Gliederung,

\section{DESAGA Katalog 70}

Die akfuelle Arbeitsunterlage für die Planung zeitgemäßer Laborausstattung enthält bereits alle ACHEMA-Neuheiten von DESAGA.

\section{DESAGA Katalog 70}

Die Informationsquelle über allein 16 verschiedene Chromatographie- und 8 verschiedene Elektrophorese-Techniken

\section{DESAGA Katalog 70}

Die vollständige Übersicht aller DESAGA Spezialitäten, wie Apparate für DünnschichtIsoelektrische-Fokussierung, Zonenschmelzen, flüssig/flüssig Vielfachextraktion, gastro-enterale Modellresorption und trägerfreie AblenkungsElektrophorese.

\section{DESAGA Katalog 70}

Ein modernes Nachschlagewerk für das Labor. Postkarte ,sendet DESAGA Katalog 70“ genügt und Sie erhalten Ihr kostenloses Exemplar zugeschickt.

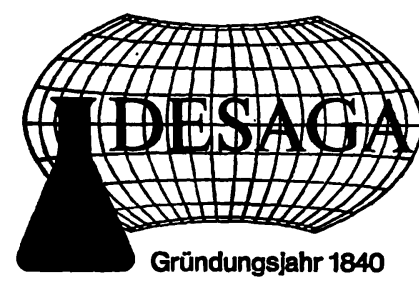

Inden Laboratorien der ganzenWelt . C.DESAGA GmbH 69 Heidelberg1 - Postfach 407 Telex 04-61736 Telefon (06221) 81013

Weltmarke der

Dünnschicht-Chromatographie und Elektrophorese 
minderung der Lactosedigestion bei nicht operierten Ratten nach Gabe von Pankreozymin in allen Fraktionen statistisch signifikant ist. Die nach subtotaler Pankreatektomie gegenüber Normaltieren festzustellende Verminderung der Lactosedigestion ohne Pankreozyminreiz ist mit Ausnahme einer Fraktion (40-50 Min. nach Perfusionsbeginn) statistisch signifikant. Nach Pankreozyminreiz ist zwischen nicht operierten Tieren und sub- total pankreatektomierten Tieren kein statistisch signifikanter Unterschied in der Lactosedigestion festzustellen. Desgleichen zeigt sich bei operierten Tieren kein Unterschied in der Lactosespaltung ohne oder nach Pankreozymingabe. In Abbildung 1 sind die Ergebnisse graphisch dargestellt.

Die Größe der Wasserexsorption ist aus Tabelle $3 \mathrm{zu}$ crsehen. Bei einem statistischen Vergleich ergibt sich

Tab. 1

Verhalten der Lactoseabsorption (\%) nach subtotaler Pankreatektomie und unter dem Einfluß von Pankreozymin

\begin{tabular}{|c|c|c|c|c|c|c|}
\hline & & \multicolumn{5}{|c|}{ Zeitraum (Min.) } \\
\hline & & $10-20$ & $20-30$ & $30-40$ & $40-50$ & $50-60$ \\
\hline $\begin{array}{l}\text { Nicht operierte Tiere } \\
\text { ohne Pankreozymin }\end{array}$ & $\overline{\mathbf{X}} \begin{array}{c}\mathbf{N} \\
(\%) \\
s \\
\mathbf{s}_{\bar{X}}\end{array}$ & $\begin{array}{l}13 \\
34,5 \\
11,3 \\
3,1\end{array}$ & $\begin{array}{l}13 \\
31,5 \\
11,6 \\
3,2\end{array}$ & $\begin{array}{l}13 \\
25,0 \\
7,8 \\
2,2\end{array}$ & $\begin{array}{l}13 \\
24,0 \\
7,3 \\
2,0\end{array}$ & $\begin{array}{r}13 \\
23,5 \\
7,2 \\
2,0\end{array}$ \\
\hline $\begin{array}{l}\text { Nicht operierte Tiere } \\
\text { nach Pankreozymin }\end{array}$ & $\overline{\mathbf{X}} \begin{array}{c}\mathbf{N} \\
\mathbf{s} \\
\mathbf{s}_{\mathbf{X}}\end{array}$ & $\begin{array}{r}7 \\
22,0 \\
12,8 \\
4,8\end{array}$ & $\begin{array}{r}7 \\
17,0 \\
10,9 \\
4,1\end{array}$ & $\begin{array}{r}6 \\
18,0 \\
6,0 \\
2,4\end{array}$ & $\begin{array}{r}6 \\
15,0 \\
7,5 \\
3,1\end{array}$ & $\begin{array}{c}6 \\
15,0 \\
7,6 \\
3,1\end{array}$ \\
\hline $\begin{array}{l}\text { Operierte Tiere } \\
\text { ohne Pankreozymin }\end{array}$ & $\overline{\mathbf{X}} \underset{\substack{N \\
\mathbf{s} \\
\mathbf{s}_{\bar{X}}}}{\mathbf{N}}$ & $\begin{array}{r}6 \\
23,5 \\
9,2 \\
3,8\end{array}$ & $\begin{array}{c}6 \\
18,0 \\
10,3 \\
4,2\end{array}$ & $\begin{array}{r}6 \\
18,5 \\
6,9 \\
2,8\end{array}$ & $\begin{array}{r}6 \\
18,0 \\
7,8 \\
3,2\end{array}$ & $\begin{array}{r}5 \\
16,0 \\
8,2 \\
3,7\end{array}$ \\
\hline $\begin{array}{l}\text { Operierte Tiere } \\
\text { nach Pankreozymin }\end{array}$ & $\overline{\mathbf{X}} \begin{array}{c}\mathbf{N} \\
(\%) \\
\mathbf{s} \\
\mathbf{s}_{\bar{X}}\end{array}$ & $\begin{array}{r}7 \\
25,0 \\
7,5 \\
2,9\end{array}$ & $\begin{array}{r}7 \\
22,0 \\
7,6 \\
2,9\end{array}$ & $\begin{array}{r}7 \\
19,5 \\
8,1 \\
3,1\end{array}$ & $\begin{array}{r}6 \\
18,5 \\
6,7 \\
2,7\end{array}$ & $\begin{array}{r}6 \\
15,5 \\
7,9 \\
3,2\end{array}$ \\
\hline
\end{tabular}

Tab. 2

Signifikanzprüfung der Lactosedigestion mit dem STUDENT-t-Test

\begin{tabular}{|c|c|c|c|c|c|}
\hline & \multicolumn{5}{|c|}{ Zeitraum (Min.) } \\
\hline & 10 p $^{20}$ & $\underset{p}{20-30}$ & ${ }_{\mathrm{p}}^{30-40}$ & $\begin{array}{c}40-50 \\
p\end{array}$ & $50 \underset{p}{60}$ \\
\hline $\begin{array}{l}\text { Nicht operierte Tiere } \\
\text { ohne Pankreozymin } \\
\text { gegen } \\
\text { nicht operierte Tiere } \\
\text { nach Pankreozymin }\end{array}$ & $<0,025$ & $<0,01$ & $<0,05$ & $<0,025$ & $<0,025$ \\
\hline $\begin{array}{l}\text { Nicht operierte Tiere } \\
\text { ohne Pankreozymin } \\
\text { gegen } \\
\text { operierte Tiere } \\
\text { ohne Pankreozymin }\end{array}$ & $<0,05$ & $<0,025$ & $<0,05$ & $<0,10$ & $<0,05$ \\
\hline $\begin{array}{l}\text { Nicht operierte Tiere } \\
\text { nach Pankreozymin } \\
\text { gegen } \\
\text { operierte Tiere } \\
\text { nach Pankreozymin }\end{array}$ & $<0,35$ & $<0,20$ & $<0,40$ & $<0,25$ & $<0,475$ \\
\hline $\begin{array}{l}\text { Operierte Tiere } \\
\text { ohne Pankreozymin } \\
\text { gegen } \\
\text { opperierte Tiere } \\
\text { nach Pankroezymin }\end{array}$ & $<0,40$ & $<0,25$ & $<0,40$ & $<0,475$ & $<0,475$ \\
\hline
\end{tabular}

Tab. 3

Wasserexsorption (\%) nach subtotaler Pankreatektomie und unter dem Einfluß von Pankreozymin

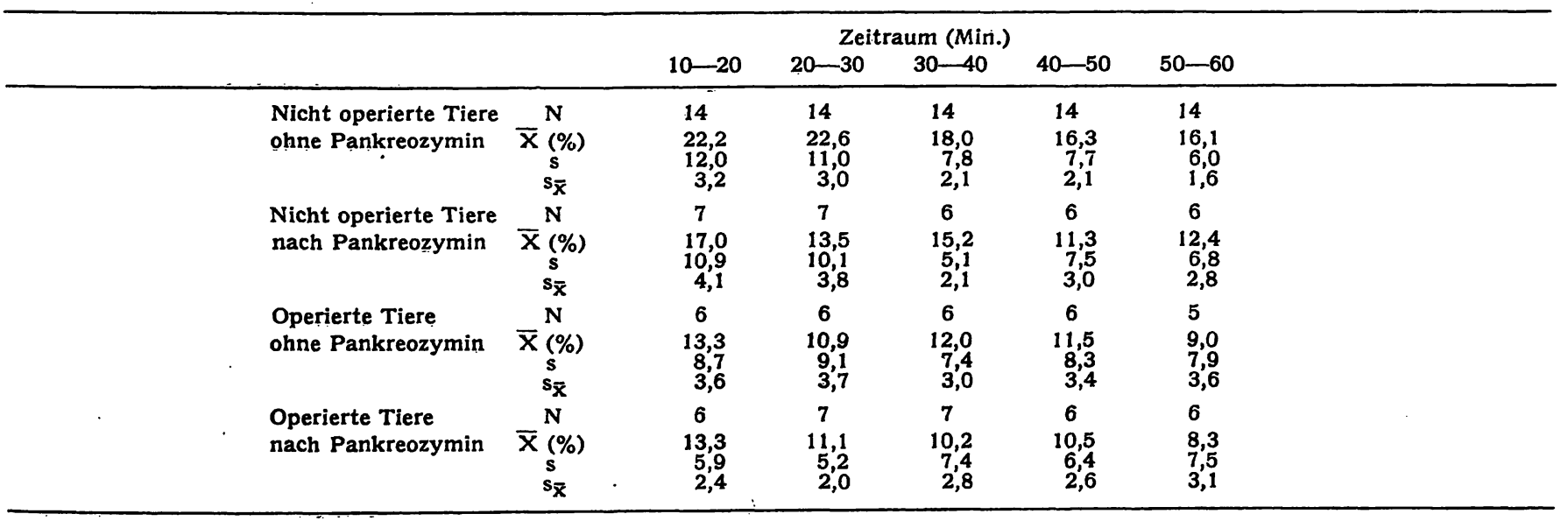


Tab. 4

Signifikanzprüfung der Wasserexsorption mit dem STUDENT-t-Test

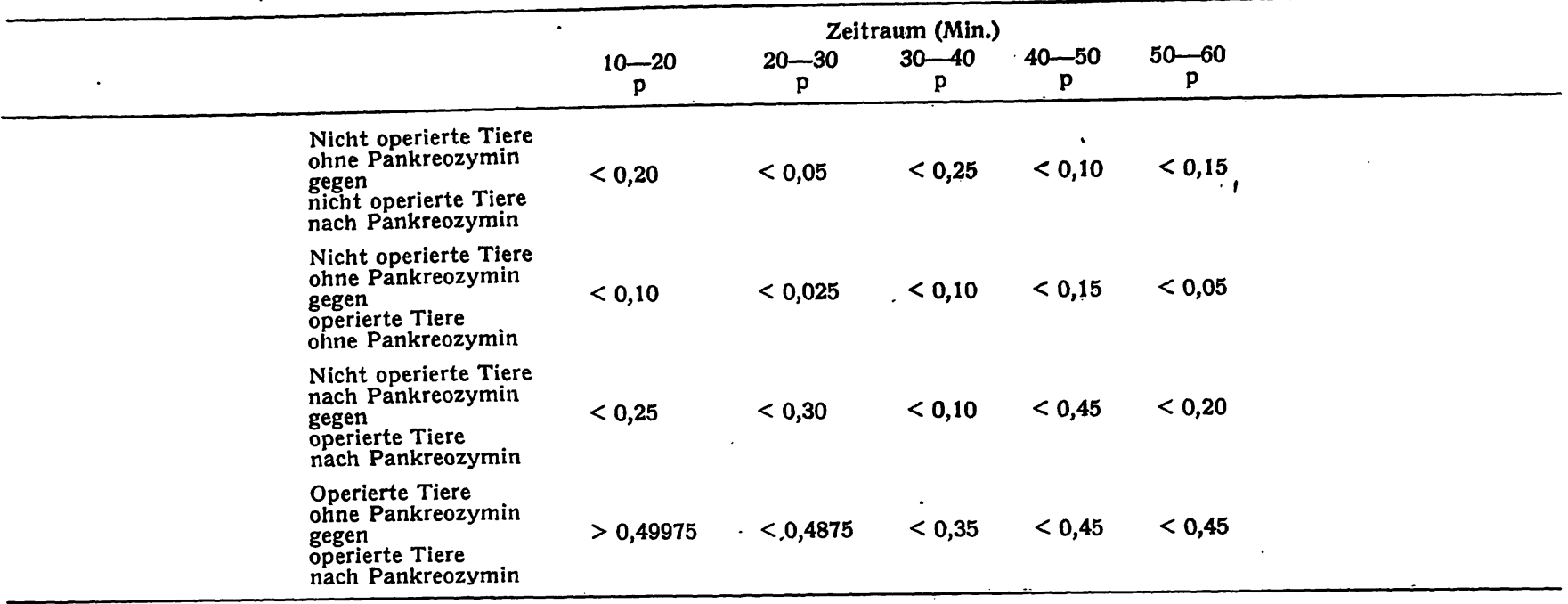

Tab. 5

Galaktosekonzentration im Perfusat (mg/100 ml)

\begin{tabular}{|c|c|c|c|c|c|c|c|}
\hline & & \multicolumn{5}{|c|}{ Zeitraum (Min.) } & \\
\hline & & $10-20$ & $20-30$ & $30-40$ & $40-50$ & $50-60$ & \\
\hline $\begin{array}{l}\text { Nicht operierte Tiere } \\
\text { ohne Pankreozymin }\end{array}$ & $\begin{array}{c}\mathrm{N} \\
\overline{\mathrm{X}}(\%) \\
\mathbf{s} \\
\mathrm{s}_{\overline{\mathrm{X}}}\end{array}$ & $\begin{array}{l}13 \\
1,57 \\
1,66 \\
0,46\end{array}$ & $\begin{array}{l}13 \\
1,30 \\
0,97 \\
0,27\end{array}$ & $\begin{array}{c}13 \\
1,35 \\
0,78 \\
0,22\end{array}$ & $\begin{array}{l}13 \\
1,30 \\
0,71 \\
0,20\end{array}$ & $\begin{array}{l}13 \\
1,41 \\
0,67 \\
0,19\end{array}$ & \\
\hline $\begin{array}{l}\text { Nicht operierte Tiere } \\
\text { nach Pankreozymin }\end{array}$ & $\begin{array}{c}\mathbf{N} \\
\overline{\mathbf{X}} \\
\mathbf{s} \\
\mathbf{s}_{\overline{\mathbf{X}}}\end{array}$ & $\begin{array}{l}7 \\
0,99 \\
0,49 \\
0,19\end{array}$ & $\begin{array}{l}7 \\
1,30 \\
0,35 \\
0,13\end{array}$ & $\begin{array}{l}6 \\
1,08 \\
0,26 \\
0,11\end{array}$ & $\begin{array}{l}6 \\
1,25 \\
0,55 \\
0,22\end{array}$ & $\begin{array}{l}6 \\
1,13 \\
0,42 \\
0,17\end{array}$ & \\
\hline $\begin{array}{l}\text { Operierte Tiere } \\
\text { ohne Pankreozymin }\end{array}$ & $\begin{array}{c}\mathbf{N} \\
\overline{\mathbf{X}}(\%) \\
\mathbf{s} \\
\mathbf{s}_{\overline{\mathbf{x}}}\end{array}$ & $\begin{array}{l}6 \\
1,98 \\
0,81 \\
0,33\end{array}$ & $\begin{array}{l}6 \\
1,35 \\
0,34 \\
0,14\end{array}$ & $\begin{array}{l}6 \\
1,08 \\
0,22 \\
0,09\end{array}$ & $\begin{array}{l}6 \\
1,15 \\
0,16 \\
0,07\end{array}$ & $\begin{array}{l}6 \\
1,20 \\
0,21 \\
0,09\end{array}$ & \\
\hline $\begin{array}{l}\text { Operierte Tiere } \\
\text { nach Pankreozymin }\end{array}$ & 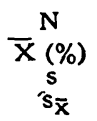 & $\begin{array}{l}7 \\
2,93 \\
2,22 \\
0,84\end{array}$ & $\begin{array}{l}7 \\
2,06 \\
1,64 \\
0,62\end{array}$ & $\begin{array}{l}7 \\
1,17 \\
1,03 \\
0,39\end{array}$ & $\begin{array}{l}6 \\
1,28 \\
0,53 \\
0,22\end{array}$ & $\begin{array}{l}6 \\
1,03 \\
0,50 \\
0,20\end{array}$ & \\
\hline
\end{tabular}

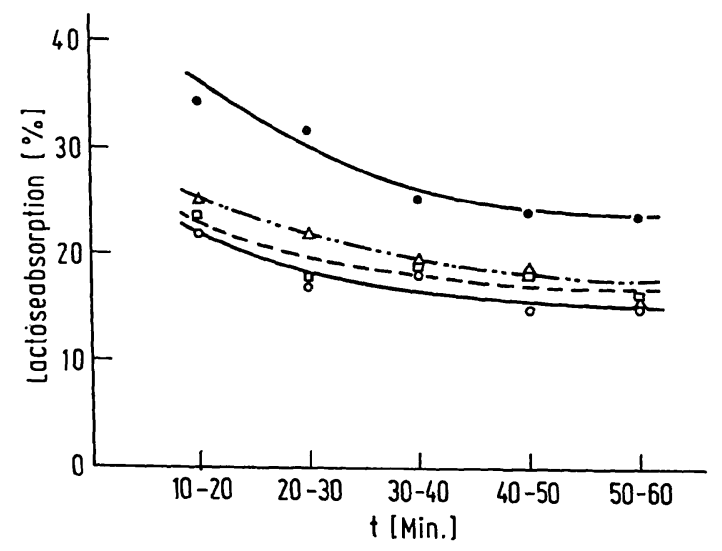

Abb. 1

Lactoseabsorption (\%)

bei Normaltieren - - -

bei Normaltieren nach Pankreozymin -o-o-

bei pankreatektomierten Tieren

bei pankreatektomierten Tieren nach Pankreozymin $-\cdots \Delta \cdots$

keine so deutliche Signifikanz wie bei der Lactosedigestion (Tab. 4). Abbildung 2 zeigt die graphische Darstellung der Wasserexsorption in den verschiedenen untersuchten Kollektiven.

Der Gehalt an freier Galaktose $(\mathrm{mg} / 100 \mathrm{ml})$ des Perfusates ist in Tabelle 5 dargestellt. In allen vier Kollek-

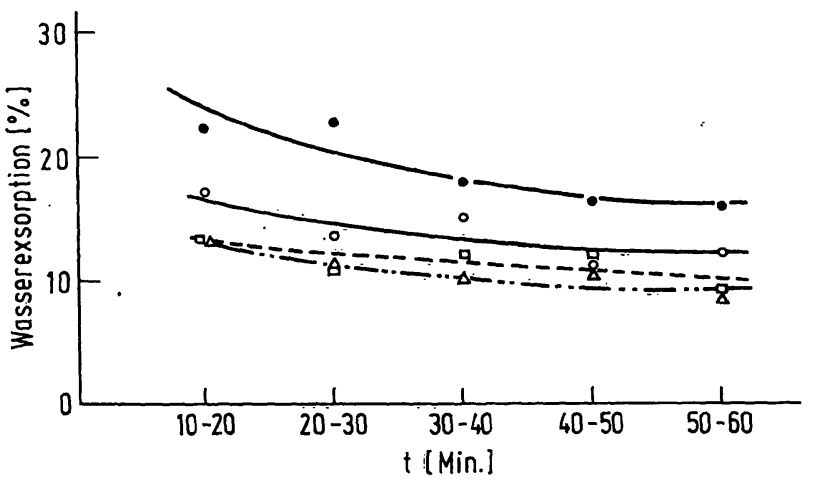

Abb. 2

Wasserexsorption (\%)

bei Normaltieren - - - -

bei Normaltieren nach Pankreozymin $-0-0$

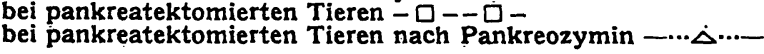

tiven liegt die Galaktosekonzentration zwischen 1 und $3 \mathrm{mg} / 100 \mathrm{ml}$. Auf einen statistischen Vergleich der Galaktosekonzentrationen wurde verzichtet, da die Streuungen in den einzelnen Kollektiven recht groß sind und die Abweichungen bei den niedrigen Konzentrationen im Streubereich der Galaktosebestimmungs- 
methode liegen. Die histologische Untersuchung der Dünndarmschleimhaut normaler und pankreatektomierter Ratten ergab keine morphologischen Veränderungen bei den operierten Tieren.

\section{Diskussion}

Subtotal pankreatektomierte Tiere verfügen noch über etwa 5\% ihres Pankreasgewebes. Einige Wochen nach der Operation zeigen männliche Tiere einen manifesten Diabetes $(6,7)$. Der Nüchternblutspiegel des immunologisch meßbaren Insulins ist gegenüber Kontrolltieren gering (nicht signifikant) vermindert, das nach subtotaler Pankreatektomie verbliebene Pankreasgewebe reicht jedoch nicht aus, um nach intravenöser Glucosebelastung einen Anstieg des immunologisch meßbaren Insulins über den Nüchternwert normaler Tiere hinaus hervorzurufen (8).

Es ist bekannt, daß Pankreozymin eine Insulinfreisetzung aus dem Pankreas hervorruft (9). Deshalb sollte die Frage untersucht werden, ob, die Hemmung der Lactosedigestion durch Pankreozymingabe bei normalen Ratten (1) ein Effekt des erhöhten Insulinspiegels oder ein direkter Pankreozymineffekt ist. Die erhobenen Befunde lassen sich jedoch nicht $z$ wanglos in ein bestehendes Modell der Lactosedigestion einordnen, sie gestatten aber, im Rahmen der bisherigen Modellvorstellungen der Zuckerdigestion und -adsorption Hypothesen aufzustellen.

Die signifikante Verminderung der Lactosespaltung nach Pankreozymingabe bei normalen, nicht operierten Tieren könnte auf den reaktiv erhöhten Insulinspiegel zurückzuführen sein. Die bei subtotal pankreatektomierten Ratten im Vergleich zu Kontrolltieren signifikant verminderte Lactoseabsorption (Tab. 2, Abb. 1) kann allerdings nicht auf einer Insulinausschüttung, sondern könnte eher auf einem Insulinmangel beruhen. Bei pankreatektomierten Tieren besteht kein Unterschied in der Lactoseverwertung ohne Pankreozymingabe oder nach intravenöser Pankreozyminapplikation. Dieser Befund könnte dadurch erklärt werden, $\mathrm{da} B$ es nach Pankreozyminreiz zu keiner Inșulinfreisetzung aus dem restlichen Pankreasgewebe kommt. Aus unseren Ergebnissen ließe sich der Schluß ziehen, daß sowohl ein Insulinmangel (z. B. nach Pankreatektomie) als auch ein Insulinüberschuß (z. B. nach Pankreozyminreiz) hemmend auf die Lactosespaltung wirken. Eine optimale Lactoseverwertung wäre demnach an einen optimalen Insulinspiegel gebunden. Bei zu niedrigem Insulinspiegel im Verhältnis zum Blutglucosespiegel könnte die Hemmung der Lactosedigestion als Schutzmechanismus vor einer zunehmenden Hyperglykämie angesehen werden.

Neben der er.wähnten Möglichkeit eines Insulineffektes muß auch ein direkter Pankreozymineffekt erörtert werden. $\mathrm{Da}$ bisher keine Methode zur Pankreozyminbestimmung im Blut bekannt ist, muß auch die folgende Erklärung hypothetisch bleiben.

Es ist denkbar, $\mathrm{da} B$ Pankreozymin und Insulin einen Gegenregulationsmechanismus bilden.

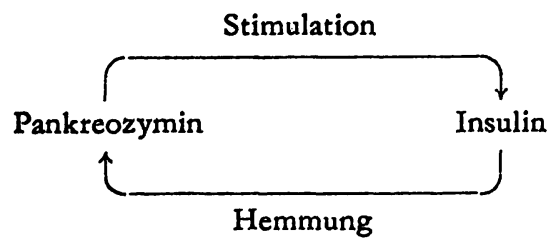

Bei pankreatektomierten, diabetischen Tieren könnte dieser Regelkreis gestört sein, da der verminderte Insulinspiegel eine verstärkte endogene Pankreozyminproduktion verursacht, die wegen Nichtansprechens des endokrinen Pankreas mit fehlendem Insulinanstieg nicht gebremst wird. Der Pankreozyminblutspiegel wäre daher erhöht.

Diese Hypothese könnte alle genannten Ergebnisse (Tab. 2 und 3) erklären:

1. Die Hemmung der Lactoseabsorption durch intravenöse Pankreozymingabe bei normalen, nicht operierten Tieren ist ein direkter Effekt des von außen zugeführten Pankreozymins.

2. Die Hemmung der Lactoseabsorption bei pankreatektomierten, diabetischen Tieren gegenüber Kontrolltieren (in beiden Fällen ohne Pankreozyminreiz) beruht auf einem erhöhten endogenen Pankreozyminspiegel, da der Regelkreis Insulin-Pankreozymin ausgefallen ist.

3. Nach intravenöser Pankreozyminstimulation besteht zwischen nicht operierten und pankreatektomierten Tieren kein signifikanter Unterschied der Lactosespaltung, gegenüber Normaltieren ohne Pankreozyminreiz ist sie jedoch in beiden Fällen vermindert. Die fehlende Beeinflussung der (ohnehin verminderten) Lactosespaltung pankreatektomierter Tiere nach Pankreozyminreiz wird dadurch erklärt, daß die Erhöhung des Pankreozyminblutspiegels über einen bestimmten Grenzwert keine verstärkte Hemmung mehr verursacht. Da bei diabetischen Tieren der Pankreozyminspiegel primär erhöht ist, ist eine weitere Zufuhr von außen unwirksam.

4. Pankreatektomierte Tiere zeigen mit und ohne intravenöse Pankreozyminzufuhr keine unterschiedliche Lactoseverwertung, da aus dem unter 3. genannten Grund die exogene Pankreozymingabe unwirksam bleibt.

Die eingangs gestellte Frage, ob es sich um einen direkten Pankreozymineffekt oder um einen Insulineffekt handelt, läßt sich durch die Ergebnisse unserer Untersuchungen noch nicht beantworten. Beide Möglichkeiten sind denkbar.

In jedem Fall stellt sich die Frage, wo der Hemmungsmechanismus lokalisiert ist, an der Lactase, am Monosacoharid-Carrier oder am System des zyklischen AMP. Bei in vitro-Versuchen am Dünndarm diabetischer Ratten zeigten OLSEN und ROSENBERG (10) eine gesteigerte intrazelluläre Akkumulation von Aminosäuren, Galaktose und 3-O-Methylglucose, unabhängig von dem den Diabetes auslösenden Agens. Da Galaktose ein Lactaseinhibitor ist (11), kann die Hemmung der Lactosedigestion bei pankreatektomierten Tieren auf einer Lactasehemmung durch ,angestaute" Galaktose in der Mukosazelle 
beruhen. Eine Hemmung des Monosaccharid-Carriers mit konsekutiver Lactasehemmung wäre aber ebenfalls möglich, da es wegen der engen räumlichen Nachbarschaft von Lactase und Carrier (12) zu einer lokalen Erhöhung der Galaktosekonzentration im Bürstensaum kommen kann ohne Vermehrung der Galaktose im Perfusat (Tab. 5). Daneben muß auch eine direkte Hemmung der Lactaseaktivität diskutiert werden ohne Beeinflussung des Carriers. Geplante in vitro-Untersuchungen der Lactaseaktivität unter den geschilderten Versuchsbedingungen werden weiteren Aufschluß über die aufgeworfenen Fragen liefern.

Der Verlauf der Kurven in Abbildung 1 zeigt mit zunehmender Perfusionsdauer eine deutliche Verminderung der Lactosedigestion mit Annäherung an einen konstanten Wert nach 60 Minuten. Dieser zunächst unerklärlich erscheinende Kurvenverlauf kann dadurch erklärt werden, daß es - auch bei Normaltieren - zu einer Akkumulation von Lactose- spaltprodukten (Glucose und Galaktose) in der Mukosazelle kommt mit sekundärer Inhibierung der Lactase. $\mathrm{Zu}$ Beginn der Perfusion enthält die Mukosazelle noch keine Produkte der gespaltenen Lactose, eine Lactasehemmung ist daher nicht anzunehmen. Mit zunehmender Perfusionsdauer ergibt sich ein immer größer werdender Galaktoseanstieg in der Zelle mit zunehmender Lactasehemmung, bis schließlich ein' Gleichgewichtszustand erreicht ist, in dem sich die Lactosespaltung im Bürstensaum und Abtransport bzw. Metabolisierung der Lactosespaltprodukte in der Mukosazelle die Waage halten.

Die Kurven der Wasserexsorption (Abb. 2) verlaufen gleichsinnig wie die der Lactosedigestion. Die Unterschiede sind jedoch nicht so deutlich signifikant wie die der Lactosedigestion (Tab. 4). Eine Verfälschung der Werte für die Nettowasserbewegung durch Pankreassekret nach Pankreozyminstimulation ist ausgeschlossen, da das perfundierte Darmstück proximal äbgebünden war.

\section{Literatur}

1. Böhmer, R., und K. RommeL, diese Z. 8, 403 (1970). - 2. SHApIro, R., und G. Pincus, Proc. Soc. Exper. Biol. Med. N. Y. 34, 416 (1936). - 3. INGLe, D. J., and G. W. ThorN, Amer. J. Physiol. 132, 670 (1941). - 4. Foglia, V. G., Rev. Soc. argent. Biol. 20, 21 (1944). - 5. Schubert, R., und H. WERNER, Zschr. exper. Med. 131, 90 (1959). - 6. Foglia, V. G., R. Borghelli, R. A. ChIERI, E. Fernandez-Collazo, J. Spinder und O. Wesely, Diabetes 12 , 231 (1963). - 7. Martin, F. H. und P. E. LACY, Diabetes 12, 328 (1963). - 8. Goberna, R., R. D. Fussgänger, S. Raptis, H. Dit-
SChuneit und E. F. Pfeiffer, Horm. Metab. Res. 1, 175 (1969) -9. UNGer, R. H., H. KetTterer, J. Dupré und A. M. Eisentraut J. Clin. Invest. 46, 630-645 (1967). - 10. Oxșon, W. A., und J. H. Rosenberg, J. Clin. Invest. 49, 96 (1970). - 11. SemenzA, G., Intestinal oligosaccharidases and disaccharidases. In: Handbook of Physiology, Vol. V (1968), p. 2543 Amer. Physiol. Sos., Washington, D, C. (1968). - 12. CRANe, R. K., Amer. J. Clin. Nutr. 22, 242 (1969).
Doz. Dr. K. Rommel Dr. R. Böhmer Dr. R. Goberna 79 Ulm (Donau) Steinhövelstr. 9 